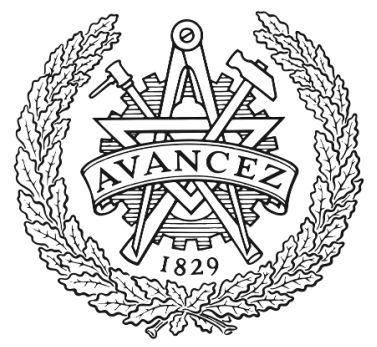

CHALMERS

UNIVERSITY OF TECHNOLOGY

\title{
Non-Thermal Absorption and Quantum Efficiency of SINIS Bolometer
}

Downloaded from: https://research.chalmers.se, 2023-04-26 01:59 UTC

Citation for the original published paper (version of record):

Tarasov, M., Gunbina, A., Yusupov, R. et al (2021). Non-Thermal Absorption and Quantum Efficiency of SINIS Bolometer. IEEE Transactions on Applied Superconductivity, 31(5). http://dx.doi.org/10.1109/TASC.2021.3057327

N.B. When citing this work, cite the original published paper.

(O2021 IEEE. Personal use of this material is permitted.

However, permission to reprint/republish this material for advertising or promotional purposes 


\title{
Non-Thermal Absorption and Quantum Efficiency of SINIS Bolometer
}

\author{
Michael Tarasov, Aleksandra Gunbina ${ }^{\circledR}$, Renat Yusupov, Artem Chekushkin, Daria Nagirnaya, Sergey Lemzyakov, \\ Vyacheslav Vdovin, Valerian Edelman, Alexei Kalaboukhov, and Dag Winkler (1)
}

\begin{abstract}
We study mechanisms of absorption in two essentially different types of superconductor-insulator-normal metalinsulator-superconductor (SINIS) bolometers with absorber directly placed on $\mathrm{Si}$ wafer and with absorber suspended above the substrate. The figure of merit for quantum photon absorption is quantum efficiency equal to the number of detected electrons for one photon. The efficiency of absorption is dramatically dependent on phonon losses to substrate and electrodes, and electron energy losses to electrodes through tunnel junctions. The maximum quantum efficiency can approach $n=h f / k T=160$ at $\mathrm{f}=350 \mathrm{GHz} \mathrm{T}$ $=0.1 \mathrm{~K}$, and current responsivity $\mathrm{d} I / \mathrm{d} P=e / k T$ in quantum gain bolometer case, contrary to photon counter mode with quantum efficiency of $\mathrm{n}=1$ and responsivity $\mathrm{d} I / \mathrm{d} P=e / h f$. In experiments, we approach intrinsic quantum efficiency up to $n=80$ electrons per photon in bolometer with suspended absorber, contrary to quantum efficiency of about one for absorber on the substrate. In the case of suspended $\mathrm{Cu}$ and $\mathrm{Pd}$ absorber, Kapitsa resistance protect from power leak to Al electrodes.
\end{abstract}

Index Terms-Bolometers, nanoelectronics, submillimeter wave integrated circuits, superconducting microwave devices.

\section{INTRODUCTION}

$\mathbf{M}$ ICROWAVE detectors of various configurations based on NIS junctions investigated since the 90 s, starting with the pioneering work [1]. The detection mechanism usually considered as bolometric [2]. This approach is applicable when the photon energy is rather low. [3]. When the signal frequency increase, the absorption mechanism becomes quantum [4].

At very low frequencies, we can apply a purely classical description, when all conduction electrons receive an energy

Manuscript received November 26, 2020; revised January 10, 2021 and January 29, 2021; accepted January 29, 2021. Date of publication February 4, 2021; date of current version March 19,2021. This work was supported in part by the Swedish Research Council (Vetenskapsrådet, project \#2016-05256), in part by the Knut and Alice Wallenberg Foundation, and in part by the Swedish Infrastructure for Micro- and Nanofabrication- Myfab. This work was carried out at the IREE RAS within the framework of the state task and RFBR 19-52-80023 project. The some of devices were fabricated in the framework of RSF project \#19-19-00499. The fabrication was carried out using USU 352529 facilities. (Corresponding author: Mikhail Tarasov.)

Michael Tarasov, Renat Yusupov, Artem Chekushkin, and Daria Nagirnaya are with the Kotelnikov Institute of Radio Engineering and Electronics, Moscow 125009, Russia (e-mail: tarasov@ hitech.cplire.ru).

Aleksandra Gunbina and Vyacheslav Vdovin are with the Institute of Applied Physics, Novgorod 603155, Russia.

Sergey Lemzyakov and Valerian Edelman are with the Kapitza Institute for Physical Problems, Moscow 119334, Russia.

Alexei Kalaboukhov and Dag Winkler are with the Chalmers University of Technology, SE41296 Göteborg, Sweden.

Color versions of one or more figures in this article are available at https: //doi.org/10.1109/TFUZZ.2020.3037972.

Digital Object Identifier 10.1109/TASC.2021.3057327 increment of the order of $e E \lambda$ where $E$ is the electric field strength and $\lambda$ the mean free path. The transition from the classical to the quantum regime considered in [5], [6]. Under the action of an alternating electric field $E=E_{0} \operatorname{Sin} \omega t$, a force $F=e E_{0} \operatorname{Sin} \omega t$ acts on an electron of mass $m$ and the equation of motion is $m \mathrm{~d}^{2} x / \mathrm{d} t^{2}=e E_{0} \operatorname{Sin} \omega t$ with a solution $x=\left(e E_{0} / m \omega^{2}\right) \operatorname{Sin} \omega t$. Pulse is $m \mathrm{~d} x / \mathrm{d} t=\left(e E_{0} / m \omega\right) \operatorname{Cos} \omega t$. Maximum of energy $E_{\max }=m v^{2} / 2$ $=\left(e E_{0}\right)^{2} /\left(2 m \omega^{2}\right)$ and average energy is $E=\left(e E_{0}\right)^{2} /\left(4 m \omega^{2}\right)$. The criterion for the transition to the quantum regime will be the equality of the energy of the quantum of this energy, i.e., $\left(e E_{0}\right)^{2} /\left(4 m \omega^{2}\right)=\hbar \omega$. From this we obtain the criterion for the frequency separating the classical and quantum absorption regions $F_{0}=(1 / 2 \pi)\left[e^{2} E^{2} /(4 m \hbar)\right]^{1 / 3}$. We can estimate the field strength with a power of $1 \mathrm{pW}$ in an absorber strip with a length of $1 \mu \mathrm{m}$ and a resistance of $50 \Omega$. We get a voltage drop of $7 \mu \mathrm{V}$, a field strength of $7 \mathrm{~V} / \mathrm{m}$, and the cutoff frequency $F_{0}=$ $0.25 \mathrm{GHz}$

The direct relaxation of the electron energy into phonons is additional relaxation channel that increases effective thermal conductivity and decreases sensitivity. The description of the mechanism of absorption and formation of the response requires calculating the collision integral and solving the kinetic equation [7]. Experimentally, the nonthermal absorption mechanism in SINIS structures with an absorber on a substrate directly observed in [8]. It was shown that, under irradiation, the electron energy distribution has a substantially nonequilibrium form, which differs from the Fermi distribution. Electrons, not having time to come to balance, make the main contribution to the additional tunneling current. In [9], the transition from the quantum gain regime to the unity gain observed when temperature increase from 0.1 to $0.3 \mathrm{~K}$. This transition happens due to the acceleration of energy relaxation processes.

\section{THERMAL AND NON-THERMAL ABSORPTION AND QUANTUM EFFICIENCY OF DETECTION}

\section{A. Thermal Absorption}

SINIS detectors often considered as classical devices, implying that the optical response is equivalent to the electrical response to thermal heating of the absorber by direct current [1], [2], [3], [10]. In practice, the electrical response at dc heating is always significantly higher than the optical response for microwave, terahertz, and IR radiation. In [11] with estimated NEP $=10^{-18}-10^{-20} \mathrm{~W} / \mathrm{Hz}^{1 / 2}$, whereas the experimental NEP $=7 * 10^{-17} \mathrm{~W} / \mathrm{Hz}^{1 / 2}$ is much higher. In [12] the self-noise of 


\section{Top view}

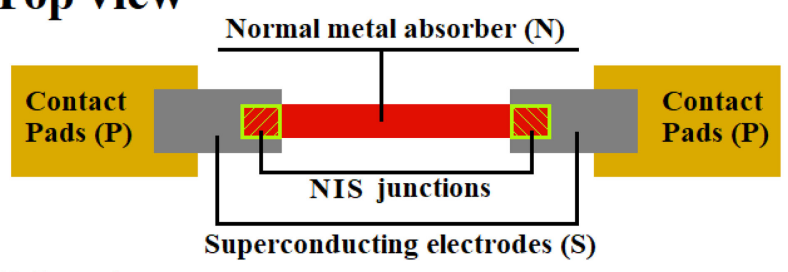

\section{Side view}

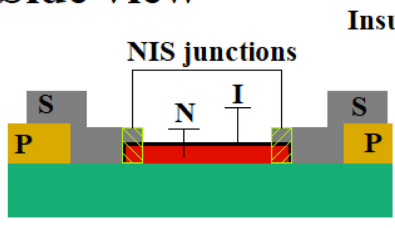

Absorber on substrate

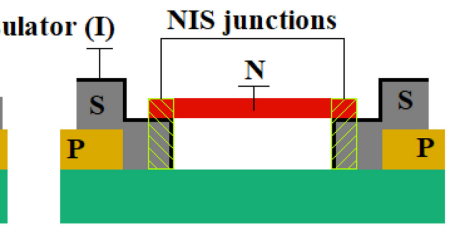

Suspended absorber
Fig. 1. Schematic image of SINIS bolometer with absorber on substrate and suspended absorber.

bolometer is estimated as $3 * 10^{-18} \mathrm{~W} / \mathrm{Hz}^{1 / 2}$, and in experiment $\mathrm{NEP}=3 * 10^{-17} \mathrm{~W} / \mathrm{Hz}^{1 / 2}$. The maximum gain for bolometer with high quantum efficiency at signal frequency of $350 \mathrm{GHz}$ can reach $n=h f / k T=160$ at $0.1 \mathrm{~K}$, and the current responsivity $\mathrm{d} I / \mathrm{d} P=e / k T=1.2 * 10^{5} \mathrm{~A} / \mathrm{W}$ in the case of a quantum gain bolometer [7] when the photon energy is thermalized to the thermal level. In contrast, the single gain mode with a quantum efficiency $\mathrm{n}=1$, corresponds to the case with one electron per photon, and the current responsivity of $\mathrm{d} I / \mathrm{d} P=e / h f=7.6 *$ $10^{2} \mathrm{~A} / \mathrm{W}$ (or $\mathrm{C} / \mathrm{J}$ ). For dc electrical heating of the absorber, the electron temperature of all conduction electrons rises; the applied power is divided by their number, therefore the current response is inversely proportional to the volume of the absorber $d I / d P=(d I / d T) /\left(5 \Sigma \nu T^{4}\right)=2.7 * 10^{5} \mathrm{~A} / \mathrm{W}$, in which $\Sigma=3$ $* 10^{9} \mathrm{~W} * \mathrm{~m}^{-3} \mathrm{~K}^{-5}$ is parameter of absorber material, $\nu=$ $10^{-20} \mathrm{~m}^{3}$ absorber volume, and $T=0.1 \mathrm{~K}$ electron temperature. In equilibrium, electron temperature determined by incoming power and electron-phonon interaction. For quantum absorption without complete thermalization of the absorbed power, many other parameters and relaxation mechanisms must be accounted.

The first prototype of the modern SINIS bolometer was the so-called Andreev bolometer consisting of a NIS thermometer and an absorber made of normal metal, directly connected to superconducting electrodes through Andreev contacts [1], [13]. In the case of Joule heating by a dc current through the Andreev contacts, a voltage response as much as $10^{9} \mathrm{~V} / \mathrm{W}$ observed in experiments. In contrast, the optical response at frequencies above $100 \mathrm{GHz}$ was only $2 * 10^{6} \mathrm{~V} / \mathrm{W}$, which is $2-3$ orders of magnitude lower than the electrical response [9]. This difference can be explained by the transparency of Andreev contacts for excited electrons with energies above the gap of aluminum $E_{q}$ $=h f>e V_{\Delta}$. This relation means that the Andreev bolometers with aluminum electrodes cannot operate at frequencies above $50 \mathrm{GHz}$.

For this reason, for bolometers in the terahertz range, NIS tunnel junctions (Fig. 1) replaced Andreev contacts. In the SINIS structure, the NIS junctions simultaneously serve as thermometers and electron coolers [14]. In such a structure, at a bath temperature of $300 \mathrm{mK}$, a decrease in the electron temperature down to $100 \mathrm{mK}$ can be obtained [15]. But such cooling with a relatively high cooling current and accompanied by additional thermal conductivity $G$, should lead to an increase in the noise equivalent power $\mathrm{NEP}^{2}=4 k T^{2} G$ in which $T$ is equilibrium temperature and $G$ is additional thermal conductivity by dc current via SINIS [16]. In the case, with different electron and phonon temperatures, the emission and absorption of phonons are uncorrelated and both processes will give an independent noise contribution [10], [17].

According to [3] the whole NEP can be estimated as $\mathrm{NEP}^{2}=$ $2 k G_{e} T_{e}{ }^{2}+2 k G_{p} T_{p}{ }^{2}=10 k \Sigma \nu\left(T_{e}{ }^{6}+T_{p}{ }^{6}\right)$, in which $T_{e}$ и $T_{p}$ are electron and phonon temperatures. Thus, even in the ideal case of a decrease in the electron temperature to zero, the total NEP cannot decrease by more than 1.4 times. Thus, cooling of only electrons is much less efficient than the simultaneous cooling of both the electron and phonon systems.

For the numerical calculation of the sensitivity, the heat balance equation is often used [1], [3], which takes into account heat sources and relaxation mechanisms $P_{s i g}+P_{b g}=\Sigma \nu\left(T_{e}{ }^{5}-\right.$ $\left.T_{p}{ }^{5}\right)+P_{\text {cool }}$, in which $P_{\text {sig }}$ is signal power, $P_{b g}$ is spurious background power, $\Sigma \nu\left(T_{e}{ }^{5}-T_{p}{ }^{5}\right)$ power flow from electron to phonon system, and $P_{\text {cool }}$ is electron cooling power. From this equation, for samples with an absorber volume of $\nu=10^{-20} \mathrm{~m}^{3}$, the voltage responsivity should be higher than $10^{9} \mathrm{~V} / \mathrm{W}$ at $300 \mathrm{mK}$ and more than $10^{10} \mathrm{~V} / \mathrm{W}$ at $200 \mathrm{mK}$. In the case of heating the absorber with a direct current or at low frequencies, responsivity values close to these estimates achieved in experiment, but at frequencies of the order of $300 \mathrm{GHz}$, the experimental results are more than an order of magnitude worse. At low resistances of NIS junctions and strong electron cooling, excited electrons are effectively removed from the absorber, their multiplication does not occur, and the current response decreases from $\mathrm{d} I / \mathrm{d} P=e / k T$ down to $\mathrm{d} I / \mathrm{d} P=e / h f$ in the case of the so-called cold electron bolometer CEB. The role of electron cooling on the response of SINIS detectors was experimentally investigated in [18], where it was shown that electron cooling does not provide the same sensitivity as the cooling of the receiver as a whole. Current quantum response efficiency for bolometer integrated in a twin-slot antenna [8] is in the range of $0.52-1.1$ for signal power $4.8-0.11 \mathrm{pW}$.

\section{B. Quantum Absorption Mechanism}

In the case of absorption of a photon by an electron with an energy much larger than the gap, for example, with a frequency of $\mathrm{f} \sim 350 \mathrm{GHz}$, the energy of an excited electron (hole) can be in the range from 0 to $h f$, i.e., reach an equivalent electron temperature up to $16 \mathrm{~K}$. The time constant of the electron-electron interaction at this temperature is much larger than the electron-phonon relaxation time. As a result, first in a time $\tau_{e p} \sim 12$ ps a phonon with energy up to $16 \mathrm{~K}$ created. For this phonon, there are three relaxation channels: escape into the substrate, escape into superconducting electrodes, or interact with an electron with time $\tau_{p e} \sim 1$ ps. In the latter case, an electron-hole pair created with characteristic energies $\pm h f / 2$ above and below the Fermi level. An excited electron with energy 
TABLE I

DyNAMICS OF 350 GHz PHOTON RELAXATION

\begin{tabular}{|c|c|c|c|c|}
\hline Process & $\begin{array}{l}\text { Equiv. } \\
\text { temp. }\end{array}$ & $\begin{array}{l}\text { Time con- } \\
\text { stant }\end{array}$ & $\begin{array}{l}\text { Number of } \\
\text { particles }\end{array}$ & $\begin{array}{l}\text { Phonon and electron } \\
\text { escape from absorber }\end{array}$ \\
\hline $\begin{array}{l}\text { el-ph } \\
\text { ph-el } \\
\text { el-el }\end{array}$ & $16 \mathrm{~K}$ & $\begin{array}{l}\tau_{e p}=12 \mathrm{ps} \\
\tau_{p e}=0.7 \mathrm{ps} \\
\tau_{e e}=0.25 \mathrm{~ns}\end{array}$ & 1 & $\begin{array}{l}\text { Transvers phonons } \\
10 \mathrm{ps}, \\
\text { Lateral phonons } 1 \mathrm{~ns}\end{array}$ \\
\hline $\begin{array}{l}\text { el-ph } \\
\text { ph-el } \\
\text { el-el }\end{array}$ & $8 \mathrm{~K}$ & $\begin{array}{l}\tau_{e p}=0.2 \mathrm{~ns} \\
\tau_{p e}=2.8 \mathrm{ps} \\
\tau_{e e}=1 \mathrm{~ns}\end{array}$ & 2 & $\begin{array}{l}\text { Transvers phonons } \\
10 \mathrm{ps} \\
\text { Lateral Phonon } 1 \mathrm{~ns}\end{array}$ \\
\hline $\begin{array}{l}\text { el-ph } \\
\text { ph-el } \\
\text { el-el }\end{array}$ & $4 \mathrm{~K}$ & $\begin{array}{l}\tau_{e p}=3.2 \mathrm{~ns} \\
\tau_{p e}=11 \mathrm{ps} \\
\tau_{e e}=4 \mathrm{~ns}\end{array}$ & 4 & $\begin{array}{l}\text { Transvers phonons } \\
10 \mathrm{ps,} \\
\text { Lateral phonons } 1 \mathrm{~ns}\end{array}$ \\
\hline $\begin{array}{l}\text { el-ph } \\
\text { ph-el } \\
\text { el-el }\end{array}$ & $2 \mathrm{~K}$ & $\begin{array}{l}\tau_{e p}=51 \mathrm{~ns} \\
\tau_{p e}=44 \mathrm{ps} \\
\tau_{e e}=16 \mathrm{~ns}\end{array}$ & 24 & $\begin{array}{l}\text { Electron escape } \\
\tau_{\sin }=40 \mathrm{~ns}\end{array}$ \\
\hline ph-el & $0.6 \mathrm{~K}$ & $\tau_{p e}=0.5 \mathrm{~ns}$ & escape & $\begin{array}{l}\text { Transvers phonons } \\
10 \mathrm{ps}\end{array}$ \\
\hline el-el & $0.3 \mathrm{k}$ & $\tau_{e e}=700 \mathrm{~ns}$ & escape & $\begin{array}{l}\text { Electron escape } \\
\tau_{\sin }=40 \mathrm{~ns}\end{array}$ \\
\hline el-el & $0.1 \mathrm{~K}$ & $\tau_{e \mathrm{e}}=6.4 \mu \mathrm{s}$ & escape & $\tau_{\mathrm{sin}}=40 \mathrm{~ns}$ \\
\hline
\end{tabular}

$h f / 2$ creates a phonon with an equivalent temperature of $8 \mathrm{~K}$, and then an electron-hole pair again created, now with energy $h f / 4$ each, or an equivalent temperature of $\sim 3 \mathrm{~K}$. At this energy and below, the time of electron-electron interaction, changing as $T^{-2}$ becomes dominant in comparison with the electron-phonon one, proportional to $T^{-4}$, energy redistributed only between electrons without noticeable generation of phonons. The process of relaxation of the energy of electrons proceeds until their characteristic times reach the tunneling time determined by the NIS parameters - the transparency of the barrier, the thickness of the normal metal film, and the diffusion rate of electrons in it. For commonly used structures, this time is of the order of tens nanoseconds.

The process of phonon escape is very important in the case of high frequencies. For transverse phonons propagating normal to absorber surface, the escape from the absorber to the substrate is $\tau_{t r}=t_{a b s} / v_{\text {sound }}=5-10 \mathrm{ps}$, and for longitudinal phonons propagating along the absorber, it can reach $\tau_{l}=l_{\text {abs }} / v_{\text {sound }}$ $=0.1-0.2 \mathrm{~ns}$ for a longitudinal size of $1 \mu \mathrm{m}$ and the speed of sound $3-5 * 10^{3} \mathrm{~m} / \mathrm{s}$. For an absorber on a substrate, $\tau_{t r}$ and a fast escape of phonons into the substrate will prevail, while for a suspended absorber that does not have contact with the substrate, only a slower process with a time constant $\tau_{l}$ remains. Moreover in the case of different materials phonons partly reflected from the boundary.

\section{Equivalent Temperatures and Escape Times}

The characteristic time scales analyzed in detail in [20], [21] and presented in Table I.

The solution of the kinetic equation for the electron distribution function and the calculation of the collision integral [7] made it possible to calculate the nonequilibrium distribution function for the rapid escape of electrons through the NIS junction in the case of strong electron cooling, as well as for the case with low cooling and low transparency of the tunnel barrier for a signal at a frequency of $1 \mathrm{THz}$ and a power of $0.1 \mathrm{pW}$. It was shown that in the case of higher resistance of junctions

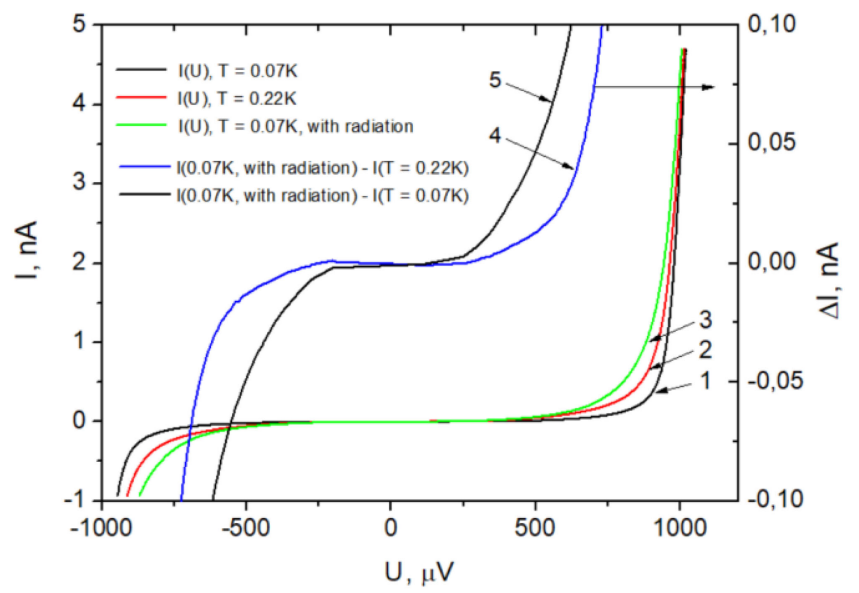

Fig. 2. IV curves for bath temperature (1) - $0.07 \mathrm{~K}$, (2)- $0.22 \mathrm{~K}$, (3)- $0.07 \mathrm{~K}$. Differences in current (4) with radiation at $0.07 \mathrm{~K}$ and without radiation at $0.22 \mathrm{~K}$, and (5) with and without radiation at $0.07 \mathrm{~K}$.

with negligible electron cooling, the level of nonequilibrium is higher and the number of detected electrons is much larger.

In addition, in [7], the detection efficiency dependence on the NIS junction resistance was calculated for various levels of unequilibrium. According to these calculations, the maximum non-equilibrium achieved at the optimal resistance of the NIS junction asymptotic resistance $\sim 5 \mathrm{k} \Omega$. In this case, the quantum efficiency reaches 30 and the current responsivity is $d I / d P=$ $30 \mathrm{e} h \mathrm{~h}$. For lower resistance values, the rate of energy escape into the electrodes is significantly higher, the thermal conductivity is higher, and excited electrons quickly leave the absorber without having time to multiply. In this case, the quantum efficiency does not exceed unity.

In conventional SINIS bolometers with NIS junction resistance less than $1 \mathrm{k} \Omega$ and an absorber on the substrate, the power loss to the substrate and superconducting electrodes can reduce the current responsivity even below the photon counter limit. In contrast to an absorber on a substrate, for a suspended absorber made of heavy metal and high-resistance NIS junctions, it is possible to realize high values of the current responsivity $e / k T$ predicted in [7] for the bolometric mode.

The effect of electron cooling at a bias voltage in the region of half the gap cannot significantly improve the response. In [14] the dependences of the electron temperature and voltage response experimentally studied at characteristic temperatures in the range of $0.1 \mathrm{~K}$ and $0.3 \mathrm{~K}$. Experimentally the expectation of significantly improve responsivity without reducing the phonon temperature of the SINIS receiver was refuted.

\section{EXPERIMENTAL RESULTS}

\section{A. Conventional Bolometer on Substrate}

IV curves and dynamic conductivity dependencies on bias voltage for 3 SINIS bolometers with $\mathrm{FeAl}$ absorber on the substrate at different temperatures and at $350 \mathrm{GHz}$ irradiation presented in Fig. 2, Fig. 3. IV curves without radiation correspond to Fermi distribution and can be correctly described 


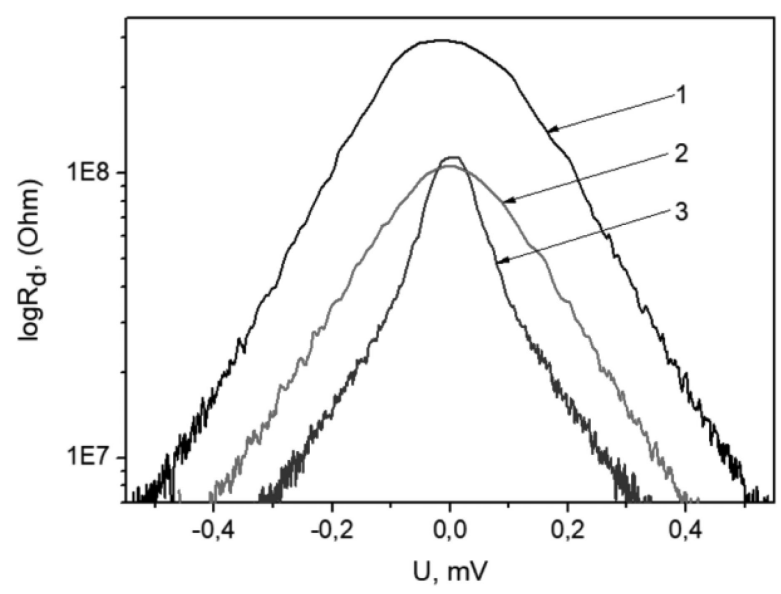

Fig. 3. Dynamic resistance (1) at $0.07 \mathrm{~K}$, (2) at $0.22 \mathrm{~K}$; and (3) at $0.07 \mathrm{~K}$ with radiation from black body source at $9 \mathrm{~K}$ with filters at $350 \mathrm{GHz}$ and bandwidth $70 \mathrm{GHz}$.

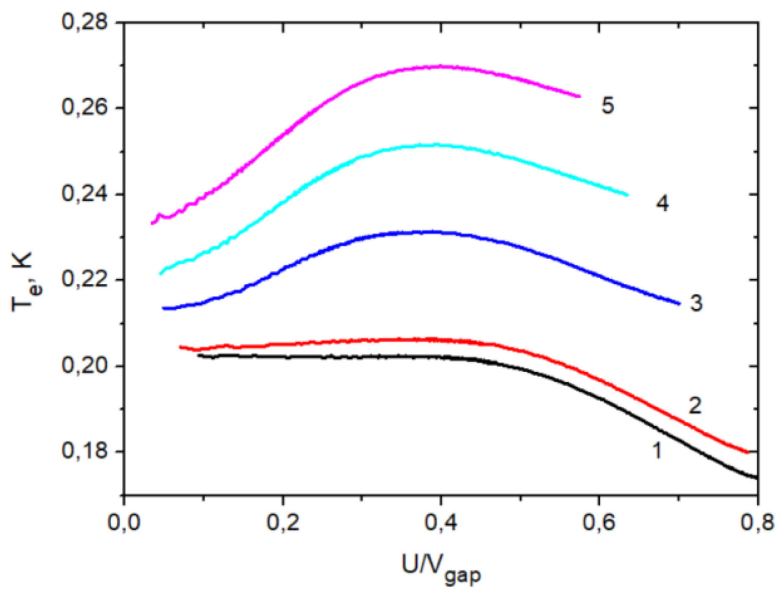

Fig. 4. Equivalent electron temperature of absorber without radiation (1), and with radiation through filter at $350 \mathrm{GHz}$ with bandwidth $70 \mathrm{GHz}$ at black body radiation source temperature of $3 \mathrm{~K}(2), 5.8 \mathrm{~K}(3), 8$ (4), and $10.5 \mathrm{~K}(5)$.

by equivalent electron temperature. Shape of curves measured at $9 \mathrm{~K}$ radiation clearly differs from equilibrium case. It is even clearer in dynamic resistance dependencies in Fig. 3. The important feature of dark curve at $220 \mathrm{mK}$ bath temperature is that the equivalent electron temperature is $235 \mathrm{mK}$. At bath temperature of $70 \mathrm{mK}$ the equivalent electron temperature is $208 \mathrm{mK}$. It means that electron system of absorber is overheated due to spurious room-temperature radiation and this device is clearly hot-electron bolometer, not a cold-electron bolometer as it was sometimes referred in literature [14], [19]. The equivalent electron temperature of absorber is presented in Fig. 4. Current quantum response efficiency for such bolometer is in the range $0.52-1.1$ for signal power $4.8-0.11 \mathrm{pW}$. Variation of electron temperature in equilibrium electron system is monotonic. Maxima in dependencies clear indicate absence of equilibrium.

\section{B. Suspended Bolometer}

We fabricated series of bolometers with absorber suspended above the substrate; see [22], [23], [24], [25]. The fabrication process is following: after first laser beam lithography patterning, a three-layer superconductor/insulator/normal metal

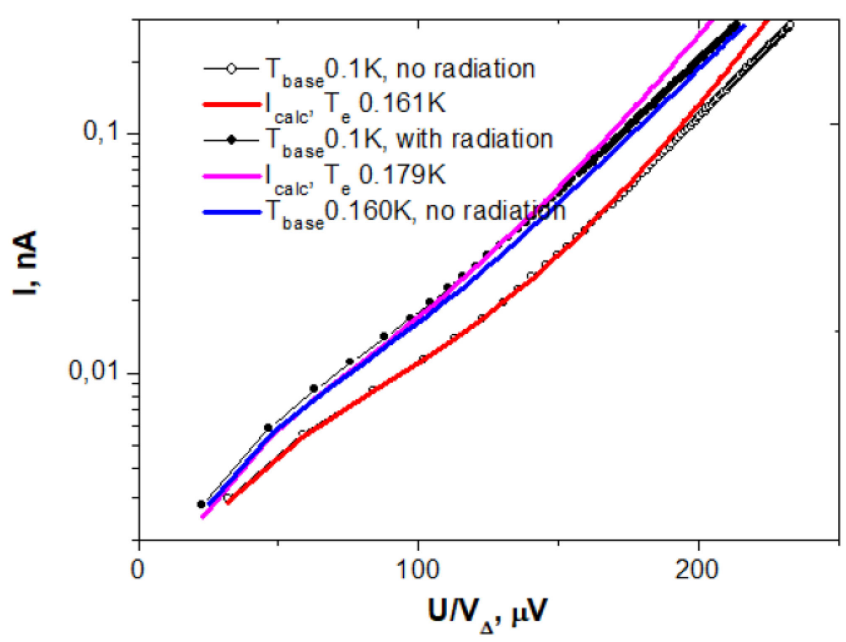

Fig. 5. IV curves of suspended Pd bolometer measured at $0.1 \mathrm{~K}$ and $0.16 \mathrm{~K}$ (red and blue), with and without radiation, and calculated for $0.179 \mathrm{~K}$ and $0.161 \mathrm{~K}$.

structure is deposited in one vacuum cycle and lifted-off. Film thicknesses are: aluminum about of $70 \mathrm{~nm}$, and normal metal $-30 \mathrm{~nm}$. In the next step, the window in resist is patterned by e-beam lithography at the area where it is intended for aluminum etching under the absorber. The aluminum etching is made in a weak alkaline solution (for example, $10 \% \mathrm{KOH}$ or photoresist developer like MF-CD-26). After that the resist is removed in acetone. In bolometer with suspended $\mathrm{Pd}, \mathrm{Cu}$, or $\mathrm{Hf}$ absorber with high-resistance NIS junctions it is possible to approach the bolometric mode with high quantum gain, see Fig. 5. In experiments, we usually measure voltage response for current bias. Maximum of response observed at voltage corresponding to about half energy gap. For asymptotic resistance of $1 \mathrm{k} \Omega$ the dynamic resistance at a half-gap bias is about $35 \mathrm{k} \Omega$ at bath temperature of $280 \mathrm{mK}$. With current response of $760 \mathrm{~A} / \mathrm{W}$, the voltage response is $2.6 * 10^{7} \mathrm{~V} / \mathrm{W}$. At bath temperature of $0.1 \mathrm{~K}$ the voltage response increase up to $1.4 * 10^{9} \mathrm{~V} / \mathrm{W}$ just due to increase of dynamic resistance.

IV curves of suspended SINIS bolometer with $\mathrm{Cu}$ absorber at $350 \mathrm{GHz}$ irradiation measured at $0.1 \mathrm{~K}$ and comparison with calculation accounting for Andreev's current according to [26] presented in Fig. 5. A clear overheating due to background radiation power is similar to [27]. Estimated by electron - phonon coupling the absorbed power according to [28] is $\mathrm{P} \approx 0.04 \pm 0.005 \mathrm{pW}\left(\approx 2 * 10^{8}\right.$ photons per second $)$. This corresponds to 80 electrons for one absorbed photon. Ultimate quantum efficiency is $n=h f / k T=160$. Mismatch of impedances and spillover losses reduce this value down to 15 for incoming radiation level.

\section{CONCLUSION}

Presented model of microwave photons non-thermal absorption and estimations of quantum efficiency in SINIS detector could be applicable for different types of cryogenic Terahertz sensors such as Transition Edge Sensors, Hot Electron Bolometers, Josephson detectors, etc. It is favorable for nonequilibrium, non-Fermi energy distribution cases, when electron temperature does not describe relaxation dynamics in the device. 


\section{REFERENCES}

[1] M. Nahum, P. L. Richards, and C. A. Mears, "Design analysis of a novel hot-electron microbolometer," IEEE Trans. Appl. Supercond., vol. 3, no. 1, pp. 2124-2127, Mar. 1993.

[2] J. Clarke, G. Hoffer, and P. Richards, "Superconducting tunnel junction bolometers," Revue De Physique Appliquée, vol. 9, pp. 69-71, 1974.

[3] D. Golubev and L. Kuzmin, "Nonequilibrium theory of a hot-electron bolometer with normal-metal-insulator-superconductor tunnel junction," J. Appl. Phys., vol. 89, pp. 6464-6472, 2001.

[4] A. Tang and P. L. Richards, "Quantum effects in the hot-electron microbolometer," IEEE TAS, vol. 5, no. 2, pp. 2599-2603, Jun. 1995.

[5] S. Rand, "Inverse bremsstrahlung with high-intensity radiation fields," Phys. Rev. B, vol. 136, no. 1B, pp. B231-B237, 1964.

[6] J. F. Seely and E. G. Harris, "Heating of a plasma by multiphoton inverse bremsstrahlung," Phys. Rev. A, vol. 7, no. 3, pp. 1064-1067, 1973.

[7] I. A. Devyatov, P. A. Krutitskii, M. Yu, and Kupriyanov, "Investigation of various operation modes of a miniature superconducting detector of microwave radiation," JETP Lett., vol. 84, no. 2, pp. 57-61, 2006.

[8] M. Tarasov et al., "Nonthermal optical response of superconductorinsulator-normal metal-insulator-superconductor tunnel structures," JETPh, vol. 119, no. 1, pp. 107-114, 2014.

[9] M. A. Tarasov, V. S. Edelman, A. B. Ermakov, S. Mahashabde, and L. S. Kuzmin, "Quantum efficiency of cold electron bolometer optical response," IEEE Trans. Terahertz Sci. Technol., vol. 5, no. 1, pp. 44-48, Jan. 2015.

[10] D. Chouvaev, D. Sandgren, M. Tarasov, and L. Kuzmin, "Optical qualification of the normal metal hot-electron microbolometer (NHEB)," in Proc. 12th Int. Symp. THz Space Technol., 2001, pp. 446-456.

[11] D. R. Schmidt et al., "A superconductor-insulator-normal metal bolometerwith microwave readout suitable for large-format arrays," Appl. Phys. Lett., vol. 86, 2005, Art. no. 053505.

[12] L. Kuzmin et al., "Photon-noise-limited cold-electron bolometer based on strong electron self-cooling for high-performance cosmology missions," Commun. Phys., vol. 2, p. 104, 2019.

[13] M. Nahum and J. M. Martinis, "Ultrasensitive-hot-electron microbolometer,"Appl. Phys. Lett, vol. 63, pp. 3075-3077, 1993.

[14] L. Kuzmin, "Ultimate cold-electron bolometer with strong electrothermal feedback," Millimeter and Submillimeter Detectors Astron. II. - Int. Soc. Opt. Photon., vol. 5498, pp. 349-362, 2004.
[15] F. Giazotto, T. T. Heikkilä, A. Luukanen, A. M. Savin, and J. P. Pekola, "Opportunities for mesoscopics in thermometry and refrigeration: Physics and applications," Rev. Mod. Phys., vol. 78, pp. 217-274, 2006.

[16] P. Richards, "Bolometers for infrared and millimeter waves," J. Appl. Phys., vol. 76, no. 1, pp. 1-24, 1994.

[17] S. R. Glowala, J. Johum, and B. Sadoulet, "Noise considerations in low resistance NIS junctions," in Proc. 7th Int. Workshop Low Temp. Detectors LTD-7, 1997, pp. 64-65.

[18] A. A. Gunbina, S. A. Lemzyakov, M. A. Tarasov, V. S. Edelman, R. A. Yusupov, "Response of a SINIS detector with electron cooling to submillimeter-wave radiation," JETP Lett., vol. 111, pp. 539-542, 2020, doi: 10.1134/S0021364020100094.

[19] L. Kuzmin, "Supersensitive cold-electron bolometers in studies of dark matter and dark energy," Uspehi Fizicheskih Nauk, vol. 48, no. 5 , pp. 519-525, 2005.

[20] M. Tarasov, V. Edelman, S. Mahashabde, and L. Kuzmin "Nonthermal optical response of superconductor-insulator-normal metal-insulatorsuperconductor tunnel structures," J. Exp. Theor. Phys., vol. 119, no. 1 pp. 107-114, 2014.

[21] M. Gershenson, D. Gong, and T. Sato, "Millisecond electron-phonon relaxation in ultrathin disordered metal films at millikelvin temperatures," Appl. Phys. Lett., vol. 79, no 13, pp. 2049-2051, 2001.

[22] R. A. Yusupov et al., "Quantum response of a bolometer based on the SINIS structure with a suspended absorber," Phys. Solid State, vol. 62 no. 9, pp. 1567-1570, 2020.

[23] M. Tarasov et al., "SINIS bolometer with a suspended absorber," J. Phys.: Conf. Ser., vol. 969, 2018, Art. no. 012088.

[24] M. Tarasov et al., "Electrical and optical properties of a bolometer with a suspended absorber and tunneling-current thermometers," Appl. Phys. Lett., vol. 110, pp. 242601, 2017, doi: 10.1063/1.4986463.

[25] M. Tarasov et al., SINIS bolometer with a suspended absorber," J. Phys.: Conf. Ser., vol. 969, 2018, Art. no. 012088.

[26] A. V. Seliverstov, M. A. Tarasov, and V. S. Edelman, The Andreev conductance in superconductor-insulator-normal metal structures," JETP, vol. 124, no. 4, pp. 643-656, 2017, doi: 10.1134/S1063776117030153.

[27] J. P. Pekola et al., "Environment-Assisted tunneling as an origin of the dynes density of states," Phys. Rev. Lett., vol. 105, 2010, Art. no. 026803.

[28] H. Courtois, F. W. J. Hekking, H. Q. Nguyen, and C. B. Winkelmann, "Electronic coolers based on superconducting tunnel junctions: Fundamentals and applications," J. Low Temp. Phys., vol. 175, pp. 799-812, 2014, doi: 10.1007/s10909-014-1101-0. 